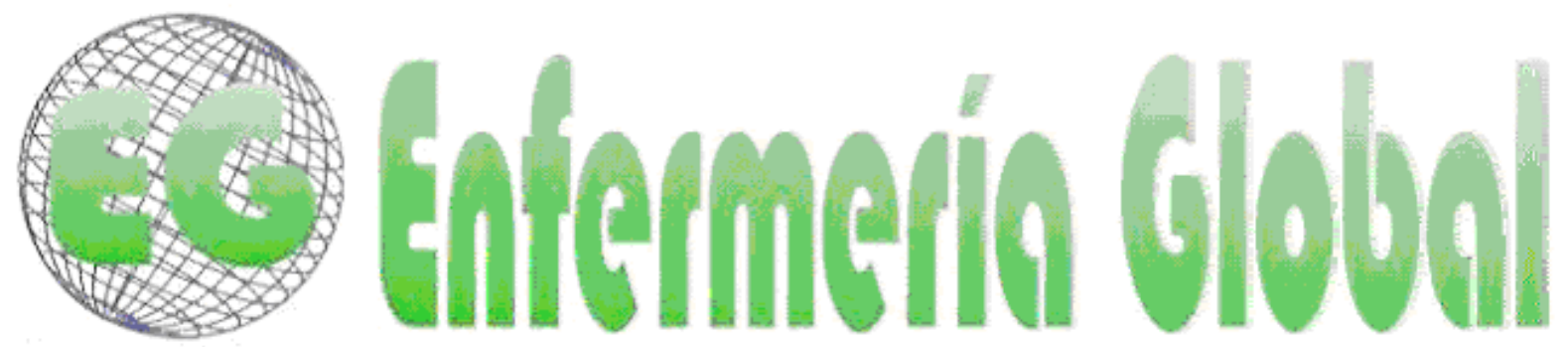

\title{
Desarrollo de la autonomía de los jóvenes portadores de espina bífida - lo que dicen los jóvenes y sus padres/cuidadores
}

Construção da Autonomia dos Jovens Portadores de Spina Bífida - O que dizem os jovens e seus pais/cuidadores

The Development of Autonomy in Young Patients with Spina Bifida - what the youngsters and their parents/careers say

\section{${ }^{*}$ Caseiro, Joel **Gonçalves, Tânia ${ }^{* * *}$ Malheiro, $M^{a}$ Isabel}

*Enfermero Generalista. Hospital Fernando Fonseca. E-mail: joel.caseiro@gmail.com ***Enfermera Generalista. Hospital do Barlavento Algarvio ${ }^{* * *}$ Profesora Adjunta. Escuela Superior de Enfermería de Lisboa. Portugal.

\author{
Palabras clave: Joven; Espina bífida; autonomia; actividades de vida diaria \\ Palavras-chave: Jovem; Spina Bífida; Autonomia; Actividades de Vida Diária \\ Keywords: Youth; Spina Bifida; Autonomy; Activities of daily living
}

\section{RESUMEN}

La Espina Bífida (EB) es una malformación congénita de la médula espinal, de acuerdo con el nivel de lesión de la médula espinal, estos niños presentan limitaciones de tipo ortopédico, urológico, motor y sensorial, que dificultan la adquisición de las habilidades necesarias para llevar a cabo sus actividades de la vida diaria (AVD) y la construcción de su autonomía. Este proceso está influenciado de forma positiva o negativa por el modo cómo los padres perciben sus necesidades. Así, los principales objetivos de este estudio fueron conocer la opinión de los jóvenes con Espina Bífida (EB) y de sus padres/cuidadores acerca del proceso de construcción de la autonomía e identificar cuáles son los factores que consideran favorables y desfavorables al mismo

A partir del análisis de las entrevistas Focus Group, a siete jóvenes con EB (Grupo A) y sus padres / cuidadores (Grupo B) surgieron las siguientes categorías: evaluación del proceso de construcción de la autonomía en general, los factores que la dificultan y los factores que fueron facilitadores y lo que sugieren para mejorar este proceso.

Los resultados revelaron que ser autónomo significa ser independiente lo que conduce a una mayor autoestima. Como factores que dificultan, destacan la actitud de los jóvenes (motivación y sentimientos de repulsa hacia su cuerpo) y de sus padres/cuidadores (superprotección). Como factores facilitadores destacan la accesibilidad, el apoyo de jóvenes con EB ya autónomos (modelos). Creemos que este estudio podrá contribuir a adecuar la intervención de enfermería y así mejorar la calidad de vida de estos jóvenes. 


\section{RESUMO}

A Spina Bífida (SB) trata-se de uma malformação congénita da espinal medula e, de acordo com o nível da lesão medular, estas crianças apresentam limitações do foro ortopédico, urológico, motor e sensorial, que dificultam a aquisição de competências, essenciais à realização das suas actividades de vida diária (AVD) e à construção da sua autonomia. Este processo é influenciado de forma positiva ou negativa pelo modo como os pais percebem as suas necessidades. Assim, os principais objectivos deste estudo foram conhecer a opinião dos jovens com Spina Bífida (SB) e de seus pais/cuidadores acerca do processo de construção da autonomia e identificar quais os factores que consideram favoráveis e desfavoráveis ao mesmo.

Com base na análise das entrevistas Focus Group realizadas a 7 jovens com SB (Grupo A) e aos seus pais/cuidadores (Grupo B) relativamente à sua opinião acerca do processo de construção da autonomia, emergiram as categorias: avaliação do processo de construção da autonomia no geral, os factores que dificultaram e os factores que foram facilitadores e o que sugerem para melhorar este processo.

Os resultados revelaram que ser autónomo significa ser independente o que conduz a uma melhor autoestima. Como factores que dificultam, destacam a atitude dos jovens (motivação e sentimentos de repulsa face ao seu corpo) e dos seus pais/cuidadores (superprotecção). Como factores facilitadores destacam a acessibilidade, o apoio de jovens com SB já autónomos (modelos). Acreditamos que este estudo poderá contribuir para adequar a intervenção de enfermagem e assim melhorar a qualidade de vida destes jovens.

\section{ABSTRACT}

The Spina Bifida (SB) is a congenital malformation of the spinal cord, according to the level of spinal cord injury, these children present limitations of the orthopedic, urological, motor and sensory forum, hindering the acquisition of skills needed to perform their activities of daily living.

This situation is extended during childhood and compromises the development of the autonomy of these young people as an essential step for its development.

The aim of this study is to know the opinion of young people with SB and their parents / caregivers, about the process of autonomy and the factors that are indicated as favorable and unfavorable to this process.

Based on the analysis of two Focus Group interviews, to seven youth with SB and their parents / caregivers, the following categories emerged: construction of autonomy in general, hygiene, mobility and elimination.

The results revealed that being autonomous means being independent and young people see this aspect as leverage in their self-esteem. The factors that relate hinder the construction of autonomy is connected to the environment: the architectural barriers, the lack of hygiene and accessibility of toilets; the parental overprotection, the lack of motivation and the feeling of revulsion against its body. As facilitating factors: accessibility of the environment, upgraded bathrooms, operational strategies and the existence of support system and a reference model. We believe that this study may be the first step to allow an appropriate intervention of the nurses resulting in an improvement of the quality of life of this youth.

\section{INTRODUCCIÓN}

La Espina Bífida (EB) es una malformación congénita que ocurre en el primer mes de gestación y que se caracteriza por el cierre incompleto del tubo neural y de los arcos vertebrales posteriores ${ }^{1}$ "(...) resultando varios niveles de alteraciones neurológicas, deformidades y compromiso de la sensibilidad de los miembros inferiores, incontinencia intestinal y vesical o retención urinaria."2 
Las necesidades específicas de los niños portadores de EB pueden variar según el nivel de lesión medular, pues es esta la que determina el compromiso neurológico y, por consiguiente, las necesidades que puedan manifestar ${ }^{3}$, a nivel de la movilidad necesitando de medios auxiliares de marcha (silla de ruedas, muletas y/o prótesis), neuro sensorial (ausencia de sensibilidad en los miembros inferiores), en el aparato genitourinario precisan sondarse $3 / 3 \mathrm{~h}$ (vejiga neurogénica) e intestinal por incontinencia de esfínter anal (uso de pañales o educación intestinal).

El número de niños con Espina Bifida que sobrevivieron hasta la fase adulta aumentó significativamente, de $10 \%$ en los años cincuenta a $90 \%$ en los ochenta, como resultado de los avances tecnológicos en el área de la salud".

Vivir con una deficiencia e incapacidad constituye un gran desafío para un adulto en la sociedade moderna actual, para el joven y su familia puede incluso convertirse en una lucha diaria, debido a las dificultades aumentadas, relacionadas con las características específicas y fragilidades de ese grupo de edad. La propia deficiencia implica alteraciones en la vida del niño, obligándolo a hacer frente a las "experiencias adversas" inherentes a los tratamientos (sucesivas hospitalizaciones) y cuidados de salud especiales (sondajes) que necesita, y que impiden o limitan las experiencias de vida normativas, deseables y facilitadoras de un desarrollo armónico.

Es imposible hablar del impacto de la deficiencia e incapacidad en la vida del joven sin hacer referencia a su proceso de desarrollo y a la fase en que se encuentra. Los desafíos relacionados con el desarrollo de cualquier joven adolescente se relacionan con busca de la identidad, autonomía emocional y económica, establecimiento de relaciones interpersonales con sus padres, la independencia de los padres y la búsqueda de una vocación. Desafíos que se hacen particularmente más difíciles de superar cuando nos referimos a los jóvenes con EB que presentan limitaciones a nivel de la movilidad, dependencia prolongada de los padres, limitaciones en las experiencias de vida, aislamiento social, pobres relaciones interpersonales con los padres $^{1}$.

Es también en este periodo cuando el niño portador de deficiencia adquiere una primera comprensión de lo que significa "ser diferente". Esta percepción, aunque pueda ser dolorosa, es esencial para su desarrollo saludable 4 . Siendo que estos adolescentes, tal como sus iguales "saludables", prefieren ser tratados apropiadamente de acuerdo con su edad y etapa de desarrollo en que se encuentran y que respeten sus capacidades y potencialidades para ser independientes y capaces de autocuidarse.

La incapacidad, siendo una situación crónica y con repercusiones en la vida del niño, es un "asunto de familia", que afectará todos los elementos, exigiendo que estos desarrollen competencias de cotejo y adaptación. De este modo, la familia puede funcionar como un elemento facilitador o, por el contrario, como un obstáculo en el proceso de adaptación ${ }^{5}$. Las dificultades diarias de los jóvenes con EB son, muchas veces, resueltas por sus padres, en un intento de protegerlos e impedir que sientan la frustración asociada al fracaso, contribuyendo este aspecto a una disminución de las oportunidades de conquistar la autonomía, considerada una de las etapas fundamentales de su desarrollo ${ }^{6}$.

En lo que concierne al joven con EB, el cuidar se extiende más allá de la gestión de sus necesidades de enfermedad. El niño con EB es un centro de actuación para la 
enfermería, donde el objetivo es alcanzar el nivel máximo de habilitación funcional teniendo en cuenta los límites del niño provenientes de la lesión y las potencialidades que presenta ${ }^{7}$. La habilitación en el niño con incapacidad física o cognitiva es un proceso de refuerzo del desarrollo, autoconcepto y autodeterminación, con el objetivo de maximizar la independencia, el bienestar físico y emocional ${ }^{1}$. El proceso de habilitación además de promover la independencia del joven, posibilita y estimula su autonomía en las actividades de vida diaria (AVD) y consecuentemente mejora su autoestima y autoconcepto.

Es fundamental el desarrollo de estos jóvenes en grupos de interayuda, el apoyo entre personas que vivencian o vivenciaon dificultades semejantes tiene efectos extremadamente positivos, en contraste con el conocimiento profesional de los prestadores de servicios. Esta estrategia es una metodología de aprendizaje eficaz para conseguir la autonomía ${ }^{8}$.

A pesar del movimiento mundial creciente en defensa de la igualdad social, especialmente en Portugal continúa existiendo un desprecio en relación a las personas que presentan algún grado o tipo de limitación en la realización de sus actividades de vida diarias. Aunque se reconozca que las personas con deficiencia o incapacidad no son un grupo homogéneo, es innegable que este constituye uno de los segmentos de la población que más ha sufrido los efectos de la exclusión social, siendo las barreras arquitectónicas la causa más evidente, impidiéndoles participar activamente en la sociedad y de ejercer su ciudadanía de una forma plena.

Y si este aspecto es una realidad en la faja de edad de los adultos, para los jóvenes que son más vulnerables la realidad es aún más dura, pues la deficiencia altera drásticamente sus vidas, estando sujetos a innúmerables situaciones adversas como tratamientos, sucesivas hospitalizaciones y cuidados de salud especiales.

La gran mayoría de los adultos con Espina Bífida tiene capacidad para ser totalmente autónomos y tener un estilo de vida muy próximo a lo "normal". El niño, el adolescente o el adulto deben estar bien equipados con competencias, de forma a alcanzar un grado de independencia, que les permita "sobrevivir" y administrar adecuadamente sus vidas en un mundo competitivo.

La intervención con el niño con EB y su familia, en el desarrollo de competencias que les permitan desempeñar las actividades de vida diaria de una forma independiente, debe iniciarse lo más precozmente posible, la elaboración de programas de intervención educativa pueden constituir la clave para minimizar la incapacidad y potencializar/maximizar las capacidades para encontrar estrategias adaptativas que le permitan un desarrollo armónico y lo más autónomo posible antes de la adolescencia ${ }^{7}$

De este modo procurar saber cuál es la opinión de los jóvenes portadores de Espina Bífida en lo relativo al proceso de construcción de la autonomía y de sus padres/cuidadores, para identificar las necesidades reales, los factores que consideran positivos y negativos en este proceso, podrá ser un punto de partida para futuras intervenciones que promuevan la funcionalidad de los niños y jóvenes con EB (lesión medular) en las actividades de vida como movilizarse, higiene, vestirse y desvestirse y eliminar, garantizando sus derechos, o sea una mayor participación en la vida social, económica y cultural de las comunidades donde se integran. 


\section{METODOLOGÍA}

Pregunta de investigación - ¿Cuál es la opinión de los jóvenes portadores de Espina Bífida en lo relativo a su proceso de construcción de la autonomía en las actividades de vida movilizarse, higiene, vestirse y desvestirse y eliminar?

Objetivo general - identificar la opinión que los jóvenes con Espina Bífida y sus padres/cuidadores tienen acerca de su proceso de autonomía en las actividades de vida eliminación, higiene personal y vestirse y movilizarse.

Participaron en este estudio 7 jóvenes portadores de Espina Bífida con edades comprendidas entre los 12 y los 18 años (grupo A) y 7 madres/cuidadores (grupo B), respectivamente. Constituyeron los criterios de selección, ser adolescente portador de Espina Bífida, tener capacidad para emitir respuestas fundamentadas, y estar vivenciando un proceso de construcción de la autonomía siendo que el Grupo B obedecía a los criterios de ser padre/madre o cuidador de los respectivos jóvenes (Grupo A). El reclutamiento fue hecho a través de la Asociación de Espina Bífida e Hidrocefalia de Portugal.

Para garantizar el cumplimiento de todos los procedimientos éticos exigidos, se pidió el consentimiento informado a los padres y a los jóvenes (de edad mayor o igual a 13 años) para integrar este estudio.

Como técnica de recogida de datos para este estudio se utilizó el "Focus Group" realizado el 19 de Marzo de 2010. El guión de la entrevista estaba compuesto por las siguientes peguntas "¿Qué significa para ustedes la autonomía? ¿Cuál es su opinión, en lo relativo al proceso de autonomía del joven portador de Espina Bífida?; ¿Cuál es su opinión en lo relativo a la autonomía de los jóvenes portadores de Espina Bífida en las actividades de la vida?; ¿Qué cree que dificulta la autonomía de los jóvenes portadores de Espina Bífida en lo relativo a las actividades de la vida? ¿Qué cree que puede facilitar la autonomía de los jóvenes portadores de Espina Bífida en las actividades de la vida?; ¿Qué creen que puede mejorar el proceso de autonomía de los jóvenes portadores de Espina Bífida en las actividades de la vida?

Limitaciones del estudio, cualquier recogida de datos cuyo abordaje se hace a un grupo supone riesgos relacionados con la naturaleza subjetiva de los sujetos, además de ser respuestas de los sujetos a la influencia de un grupo de variables que en principio no son tenidas en cuenta. Al elegir como principal metodología de recogida de datos el Focus Group, somos conscientes de un riesgo añadido, por la influencia que las ideas de unos elementos tienen en los otros, en el contexto de una entrevista de grupo.

Procurar saber, a través de los propios jóvenes con EB, sus opiniones sobre su autonomía en AVD's donde, generalmente, presentan grandes dificultades. La posibilidad de los jóvenes de responder de forma ambigua o poco profunda a las cuestiones presentadas, pudiendo también sentir alguna inhibición en la discusión de tópicos relacionados con su intimidad, constituye también una limitación a este estudio.

Como técnica de análisis de los datos, se hizo un abordaje cualitativo, tal como el análisis de contenido temático y categorial. Con algunas particularidades que los autores de referencia aconsejan por el hecho de ser una entrevista en grupo ${ }^{9,10,11 .}$ 
El corpus de análisis lo constituían las transcripciones integras de los dos focus groups. La parrilla de análisis partió en una primera fase, de guión orientador de la entrevista siendo construida y reconstruida a través de lecturas verticales de las que emergieron las categorías, subcategorías e indicadores que fueron categorizados, interpretados y que sirven de soporte al modelo de análisis y a la discusión de los resultados.

La codificación, denominada "codificacióno axial"9 presuponía retirar las diversas unidades de registro y respectiva contextualización para formar una unidad de análisis "coherente" denominada "unidades de significación" 3 . Codificación que constituye un proceso de clasificación de temas que son representados por conjuntos de datos ${ }^{12}$. En esta perspectiva los códigos son solo los significantes de las ideas constituyendo las categorias analíticas, una clasificación que el investigador utilizó para transmitir una determinada idea. De este modo, todo el trabajo de codificación y de comparación sistemática implicó perfeccionar una parrilla de análisis definitiva, donde emergieron las siguientes categorías: Actividad de vida en general, en la Higiene Personal y Vestuario, en la Movilidad y la Eliminación, como subcategorías los factores que dificultan, los factores facilitadores y lo que sugieren para mejorar este proceso. (Cuadro I).

Cuadro I -Parrilla de Análisis

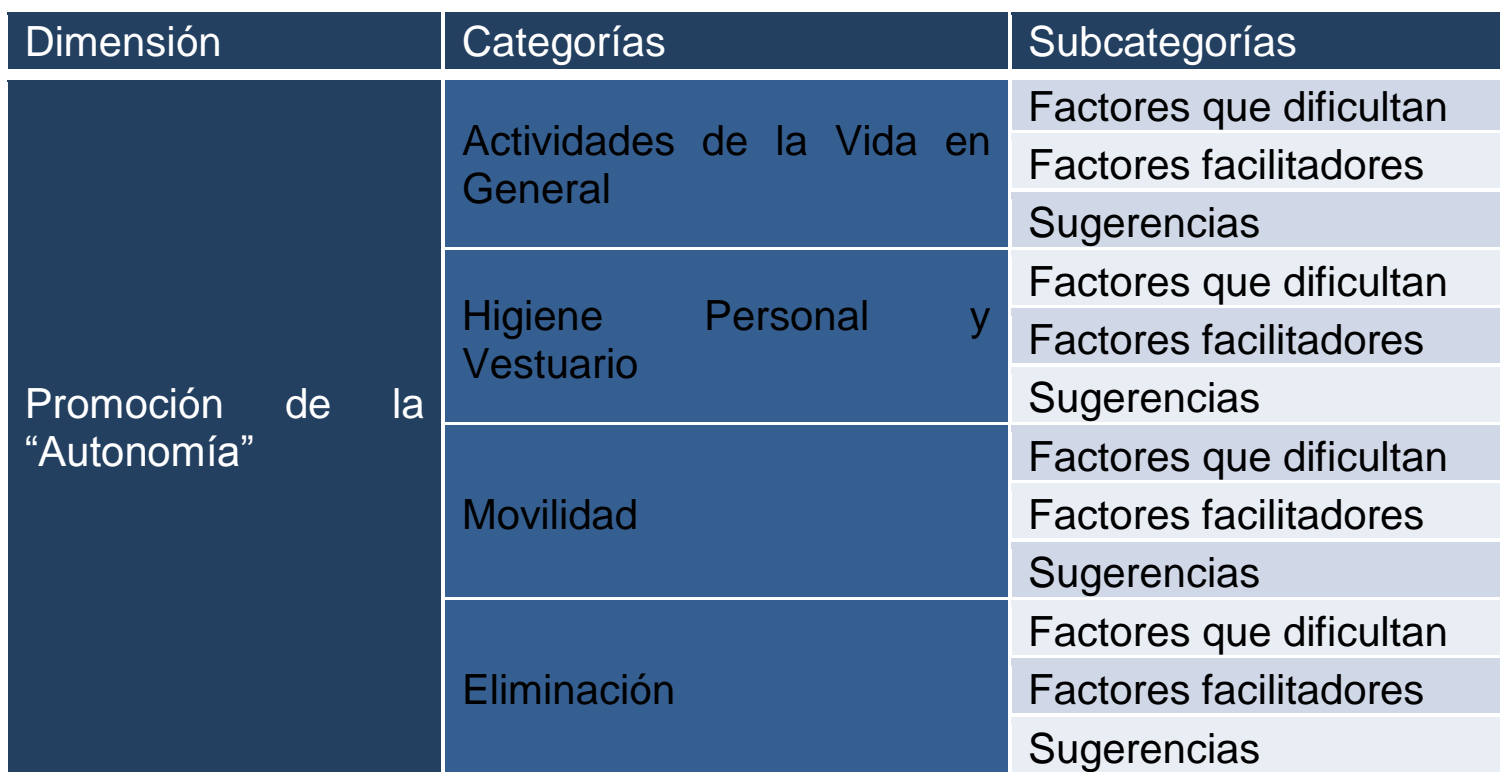

Seguidamente se hará la presentación de los resultados y un análisis global, y siempre que los resultados lo justifiquen en términos de discrepancia y/o divergencia, haremos un análisis comparativo entre los grupos.

\section{PRESENTACIÓN DE LOS RESULTADOS Y DISCUSIÓN}

En lo que se refiere al significado del "Concepto de Autonomía", para ambos grupos, está directamente relacionado con el ser independiente, considerando que una persona independiente es aquella que no depende de otra:

"yo puedo ir a cualquier lado sola... Tengo mi maleta y ya no dependo de nadie" B7. 
Resultados que coinciden parcialmente con el concepto de autonomía, que abarca las tres siguientes dimensiones; la independencia, la autodeterminación y el autocuidado, sin embargo a través del discurso de estos jóvenes podemos constatar que la dimensión independencia fue evidenciada ${ }^{13}$.

En cuanto a la opinión de los participantes sobre la "Autonomía", ambos grupos consideran posible al joven con EB ser autónomo:

"Se necesita un poco más de tiempo (...) pero todo el mundo consigue tener autonomía." A3

Para los padres/cuidadores, esa autonomía es posible desde que los jóvenes demuestran una actitud de responsabilidad, relacionada con su proceso de maduración:

“Ellos consiguem ser autónomos. Desde que tienen buena cabeza.” B2

Por consiguiente, los jóvenes portadores de EB refieren que cuanto mayor es la responsabilidad atribuida, mayor será su sentimiento de competencia en aquello que conseguirán hacer, aumentando así su autoestima:

“(..) si nos estimulamos conseguimos hacer más de lo que pensamos que conseguimos. Aumenta la autoestima (...)” A6.

Cuando la autoestima aumenta, motiva la ejecución de actividades que exigen mayor esfuerzo $^{14}$

"(...) es por las pequeñas cosas que comenzamos a ver mejor que somos capaces." A5

Cuadro II - Categoría "Actividades de la Vida en General”

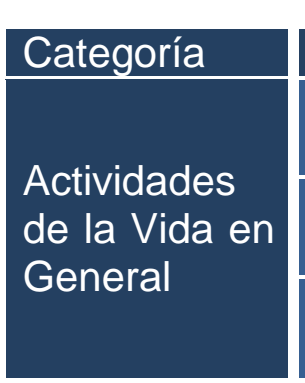

\begin{tabular}{l}
\hline Subcategorías \\
\hline $\begin{array}{l}\text { Factores que } \\
\text { dificultan }\end{array}$ \\
\hline $\begin{array}{l}\text { Factores } \\
\text { facilitadores }\end{array}$ \\
\hline Sugerencias
\end{tabular}

\section{Grupo B}

Indicadores

Frecuencia

Actitud de superprotección 21

Sistemas de soporte

Modelos de referencia 9

Refuerzo de la autoestima

17

16

En relación a la categoría "Actividades de la Vida en General", como podemos verificar en el Cuadro II, el grupo B refiere que las actitudes de superprotección por parte de los padres/cuidadores constituyen uno de los factores que influyen de forma negativa en el proceso de autonomía de los jóveness con EB:

"Nosotros los padres tenemos mucha culpa en esa parte...perjudicamos (...)" B2

“Yo, en mi caso, superprotegí a A7. (...) Y me arrepentí mucho (...)”B1. 
Resultado que demuestra la tendencia que los padres de los jóvenes con EB presentan para desarrollar un sentimiento de superprotección en relación a sus hijos, pues los consideran más vulnerables, debido a su condición de salud. La ocurrencia del fenómeno Miscarried Helping es frecuente, porque hay un desajuste de los padres en su nivel de protección y el proceso de desarrollo de los hijos, acabando por interferir en su proceso de adquisición de autonomía ${ }^{15}$.

Ambos grupos evidencian también una mayor superprotección por parte de los abuelos, que asumieron el papel de cuidadores de estos jóvenes, intentando llenar la ausencia o menor atención por parte de los padres.

En cuanto a los factores facilitadores para la misma categoría, los padres/cuidadores evidencian el apoyo proveniente de los sistemas de soporte, destacando la importancia del papel de los profesionales de educación, así como de los profesionales de salud, principalmente en la adquisición de las competencias necesarias al joven para la realización de la AVD eliminación. Consideran también, indispensable la presencia de una persona de referencia para apoyar al joven y a la familia en los momentos de crisis, destacan los profesionales de salud, de educación o simplemente una persona con experiencia en este tipo de situciones:

"Es bueno que nosotros sintamos (...) que tenemos esas personas al otro lado, cuando nos sentimos afligidos (...)"B7.

Estos resultados evidencian el papel del enfermero, que tiene una gran responsabilidad en la promoción del desarrollo de los jóvenes, especialmente en lo que dice sobre las competencias necesarias para la realización de las AVD's, y que habitualmente lo hacen en colaboración con otros técnicos de salud en el área de la rehabilitación ${ }^{16}$. Este soporte debe ser dirigido no sólo al joven sino también a su familia, para que esta pueda tomar decisiones informadas sobre las intervenciones y el apoyo proporcionado ${ }^{1}$.

Como sugerencias, el grupo B señala la utilización de modelos de referencia para reforzar la autoestima de los jóvenes portadores de EB, facilitando así el desempeño de sus AVD's. Según los padres/cuidadores, estos modelos de referencia pueden ser personas conocidas en la comunicación social o personas presentes en la red social del joven que, a pesar de presentar limitaciones físicas semejantes, conseguirá tener éxito en la vida.

Cuadro III - Categoría "Higiene Personal"

\begin{tabular}{|c|c|c|c|c|c|}
\hline \multirow{2}{*}{ Categoría } & \multirow[b]{2}{*}{ Subcategorías } & \multicolumn{2}{|l|}{ Grupo A } & \multicolumn{2}{|l|}{ Grupo B } \\
\hline & & Indicadores & Frecuencia & Indicadores & Frecuencia \\
\hline \multirow{3}{*}{$\begin{array}{l}\text { Higiene } \\
\text { Personal }\end{array}$} & $\begin{array}{l}\text { Factores que } \\
\text { dificultan }\end{array}$ & $\begin{array}{l}\text { Miedo a } \\
\text { caída }\end{array}$ & 6 & $\begin{array}{l}\text { Falta de } \\
\text { motivación }\end{array}$ & 18 \\
\hline & $\begin{array}{l}\text { Factores } \\
\text { facilitadores }\end{array}$ & & --- & $\begin{array}{l}\text { Aseos } \\
\text { adaptados }\end{array}$ & 5 \\
\hline & Sugerencias & $\begin{array}{l}\text { Aseos } \\
\text { adaptados }\end{array}$ & 3 & ------- & --- \\
\hline
\end{tabular}


En cuanto a la higiene personal, el miedo a caer_en los aseos es considerado por los jóvenes portadores de EB uno de los factores que más dificulta el desempeño de esta AVD:

"Para ir al baño (...) preciso solo de ayuda para salir de la bañera, por una cuestión de seguridad, porque he tenido varias caídas y tengo miedo a caer, (...) A6. (Cuadro III).

Es posible analizar y justificar este gran recelo, teniendo en cuenta las alteraciones a nivel sensorio-motor y ortopédico que estos jóvenes presentan y que pueden dificultar la materialización de las principales habilidades consideradas esenciales, para una mayor independencia en su autocuidado ${ }^{4}$. Del mismo modo, otros problemas derivados de su lesión, como la dificultad en la percepción visual-espacial, puede perjudicar su percepción espacial real, en el momento de la transferencia de la silla de ruedas al inodoro y viceversa, pudiendo originar una caída ${ }^{1}$.

A su vez, los padres/cuidadores destacan la falta de motivación de los jóvenes portadores de EB, asociando este factor a la desmotivación característica de los jóvenes en la adolescencia:

"(...) no importa si no se lava del todo bien, pero él también (es)tá en esa fase, de la edad, de no preocuparle mucho la higiene." B4

Para esta categoría, solo los padres/cuidadores hacen referencia a factores facilitadores, destacando las ventajas de los aseos adaptados en el domicilio:

“Desde que tiene los accesos él se pasa perfectamente al inodoro (...)"B2

El hecho de tener material adaptado constituye, según los miembros del grupo $\mathrm{B}$, un aspecto facilitador de la autonomía del joven portador de EB en la actividad de vida Higiene Personal y Vestirse.

"El A3 se asea solo, sentado en un taburete (...) B4

La adaptación de los aseos es también la sugerencia más destacada por los jóvenes portadores de EB:

“ (...) poner aseos adaptados, como por ejemplo, en los aseos públicos para deficientes, hay siempre dos cosas (barras) a cada lado del inodoro para asegurarnos, podian intentar (...) probar alguna cosa que nos ayudase a salir y entrar del aseo"A6.

Podemos constatar, que el que existan adaptaciones o material adaptado junto al joven o como recurso del joven portador de EB es favorable para que este se vuelva más autónomo o sea, más funcional. Si el joven presenta capacidades para la ejecución de las actividades, incluso con adaptaciones o medios auxiliares, se afirma que este tiene habilidad, o sea, es funcional. ${ }^{4}$ 


\begin{tabular}{|l|l|l|l|l|l|}
\hline \multirow{2}{*}{ Categoría } & Gubcategorías & Indicadores & Frecuencia & Indicadores & Frecuencia \\
\hline \multirow{3}{*}{ Movilidad } & $\begin{array}{l}\text { Factores que } \\
\text { dificultan }\end{array}$ & $\begin{array}{l}\text { Barreras } \\
\text { arquitectónicas }\end{array}$ & 18 & $\begin{array}{l}\text { Barreras } \\
\text { arquitectónicas }\end{array}$ & 34 \\
\cline { 2 - 6 } & $\begin{array}{l}\text { Factores } \\
\text { facilitadores }\end{array}$ & $\begin{array}{l}\text { Accesibilidad } \\
\text { Estrategias } \\
\text { operacionales }\end{array}$ & 2 & Accesibilidad & 11 \\
\cline { 2 - 6 } & Sugerencias & Mejora de las condiciones de accesibilidad & \\
\end{tabular}

En relación a la movilidad, el indicador barreras arquitectónicas es el factor que dificulta la autonomía más evidenciado como podemos comprobar por la frecuencia en ambos grupos (Cuadro IV). Estas barreras están presentes en la mayoría de los edificios, espacios, transportes públicos y en las escuelas.

"El (...) no es tan autónomo porque no lo dejan ser. (...) No hay accesos (....) Empieza justo en la puerta. (...) en la escuela. Transportes públicos.” B2.

Se evidencian también las implicaciones negativas de las barreras arquitectónicas a nivel de la interacción del joven con EB con sus semejantes, en una fase en que el establecimiento de relaciones interpersonales y el desarrollo de competencias sociales es tan importante para los jóvenes como la adolescencia.

"Ella tiene varios amigos y ha de hacer una selección, sólo puede ir a casa de los amigos que viven en planta baja." B1

Conociendo que la incapacidad cuando está asociada a la deficiencia física puede restringir la capacidad del joven con EB para interactuar con el ambiente envolvente, esta situación puede limitar su socialización y consecuentemente llevar al aislamiento y soledad que estos jóvenes habitualmente experimentan ${ }^{2}$.

En cuanto a los factores facilitadores, ambos grupos refieren la adopción de estrategias operacionales, principalmente la utilización de medios auxiliares de marcha:

"En la escuela uso una silla de ruedas, para andar más fácil" $A 2$.

Destacan también la accesibilidad como un factor facilitador para la movilidad:

“(...) se tuviera rampas yo consigo." A4

En cuanto a las Sugerencias que hacen, tanto los padres/cuidadores como los jóvenes portadores de EB para mejorar este proceso de construción de la autonomía, destaca la mejora de las condiciones de accesibilidad:

“ (...) cuando hubiera escaleras, colocar una rampa de lado y el pasamanos en la propia escalera, para que la persona se apoye cuando va a subir las escaleras" A5 


\begin{tabular}{|c|c|c|c|c|c|}
\hline \multirow{2}{*}{ Categoía } & \multirow[b]{2}{*}{ Subcategorías } & \multicolumn{2}{|l|}{ Grupo A } & \multicolumn{2}{|l|}{ Grupo B } \\
\hline & & Indicadores & Frec & Indicadores & Frec \\
\hline \multirow{4}{*}{ Eliminación } & \multirow{2}{*}{$\begin{array}{l}\text { Factores que } \\
\text { dificultan }\end{array}$} & \multirow{2}{*}{$\begin{array}{l}\text { Falta de higiene } \\
\text { y accesibilidad } \\
\text { en los aseos }\end{array}$} & \multirow[t]{2}{*}{18} & $\begin{array}{l}\text { Actitud de } \\
\text { superprotección }\end{array}$ & 14 \\
\hline & & & & Repugnancia & 12 \\
\hline & $\begin{array}{l}\text { Factores } \\
\text { facilitadores }\end{array}$ & $\begin{array}{l}\text { Aseos } \\
\text { adaptados }\end{array}$ & 2 & $\begin{array}{ll}\text { Modelo de } & \text { de } \\
\text { referencia } & \\
\text { portadores EB } & \end{array}$ & 20 \\
\hline & Sugerencias & $\begin{array}{l}\text { Mejora de las } \\
\text { condiciones de } \\
\text { higiene y } \\
\text { accesibilidad en } \\
\text { los aseos }\end{array}$ & 6 & $\begin{array}{l}\text { Articulación con la } \\
\text { escuela } \\
\text { (salud/asociación) }\end{array}$ & 16 \\
\hline
\end{tabular}

En lo que se refiere a eliminación, como podemos observar en el Cuadro $\mathrm{V}$, el factor que dificulta esta AVD con mayor destaque, según los jóvenes portadores de EB, es la falta de higiene y accesibilidad en los aseos, especialmente en los espacios públicos y escuelas:

"Cuando voy a las visitas de estudio, (...) a veces no hay aseos para deficientes, y yo sólo hago el sondaje cuando llego al destino." A4;

\section{“(...) todos los aseos estaban sucios.” A3.}

Sin embargo, para los padres/cuidadores el asco en tocar los genitales/heces por parte de los jóvenes portadores de EB constituye un factor que dificulta la autonomía en esta actividad. Este sentimento se manifesta principalmente en la realización del autosondaje, así como en la eliminación intestinal:

\section{"(...) no tiene autonomía alguna (...) se interrumpe porque le da mucho asco (...)" B4}

El indicador actitudes de superprotección fue también destacado por los padres/cuidadores como una actitud que dificulta el desarrollo de la autonomía en esta AVD:

“(...) he fallado en esto, lo superprotegí mucho en la parte de la autonomía de la higiene (de las heces) (...) Y lo hago por él (...)"B4

En cuanto a los factores facilitadores para la adquisición de la autonomía en la AVD "Eliminación", los jóvenes portadores de EB destacan nuevamente la existencia de aseos adaptados:

"Es más fácil. Porque en casa, tengo un aseo grande y prácticamente sólo soy yo quien va a él y en la escuela también." $A 7$

Como se comprobó, uno de los mayores problemas identificados en esta situación es el hecho de que, muchas veces, no existe un espacio apropiado en las escuelas para que el joven con EB pueda realizar el vaciamiento vesical ${ }^{1}$. 
Sin embargo para los padres/cuidadores, el apoyo de los modelos de referencia portadores de EB es el indicador más emergente:

"Entonces el hecho de ser una persona que también sintió en la piel la necesidad de adaptarse, y la experiencia de esa persona ayudó a mi hija a superar. Por tanto, es muy bueno tener (...) Una referencia, con el mismo problema." B1

Resultados que realzan la importancia del apoyo entre personas que pasaron o pasan dificultades semejantes para la construcción de la autonomía, quedan más motivadas en hacer algo que alguien semejante, o con la misma condición, consiguió hacer. La utilización de pares en los programas de educación para la autogestión, ha demostrado ser eficaz ${ }^{8}$.

Finalmente, como sugerencia, los jóvenes portadores de EB destacan la mejoría de las condiciones de higiene y de accesibilidad en los aseos:

“(...) al menos que estén limpios, para disminuir el riesgo de infecciones.” A6;

“(...) tal vez debía haber más apoyo (...) en todos los sitios a donde vamos (...) aseos grandes para meter una silla de ruedas. Es una cosa que debía de tener mayor inversión, que (...) es un bien para nuestra salud. "A5

En cuanto a los padres/cuidadores, emerge el deseo de una mejor articulación entre la escuela y las instituciones de salud y la Asociación de Espina Bifida e Hidrocefalia de Portugal:

"La salud debía de estar ligada a la educación. ¡Siempre! (...) creo que es muy importante la parte de enfermería, la parte médica (...) en la escuela.” B1

Los niños con necesidades de salud especiales presentan complejos desafíos a los profesionales de salud y de educación, lo que exige reuniones entre los profesionales, a fin de procurar en conjunto respuestas adecuadas que les permitan un desarrollo armónico basado en sus capacidades y potencialidades, y no centrado en las incapacidades y limitaciones, garantizando de este modo las condiciones para su éxito educativo ${ }^{3}$.

La articulación debe ampliarse a los restantes servicios de salud especializados que acompañan a los jóvenes con limitaciones en lo que concierne a su situación de salud. Este hecho demuestra la necesidad de los profesionales de educación de estar informados de la situación clínica del joven portador de EB, así como de los cuidados de salud que necesitan, siempre con el propósito de la continuidad en la satisfacción de sus necesidades especiales de salud (aseos adaptados, tiempo extra para realización del autosondaje, etc.) ${ }^{3}$.

\section{CONCLUSIÓN}

Teniendo en cuenta la pregunta de investigación así como los objetivos establecidos en este estudio, se puede afirmar que de un modo general estos fueron alcanzados. La heterogeneidad de los grupos encausados volvió todo el proceso de investigación desafiante y más rico, pue permitió un contacto con perspectivas diferentes entre sí, que originaron diferentes indicadores, siendo que algunas de esas perspectivas 
acabaron por cruzarse, completándose y permitieron una visión más global de este fenómeno.

En lo que se refiere al significado de autonomía, los participantes fueron unánimes, destacando que ser autónomo está directamente relacionado con ser independiente. Los jóvenes, por su parte, manifiestan un gran deseo de no depender de nadie, pues señalan que esa libertad les traerá una baza en el área de las relaciones sociales.

La conquista de autonomía es considerada fundamental para mejorar su autoestima, y motivación para la realización de tareas más complejas. Los comportamientos promotores de salud también se muestran como fundamentales en este proceso de construción de la autonomía, a pesar de que este aspecto pueda estar relacionado con la madurez intelectual de los jóvenes, el enfermero debe actuar como un agente promotor de comportamientos saludables.

Las actitudes de superprotección constituyen un factor que dificulta la adquisición de la autonomía de los jóvenes en la mayoría de las AVD's, justificando que al querer ayudar y de algun modo proteger a los hijos de frustraciones y fracasos, reconocen que ejercen una influencia negativa en este proceso. Destacar que estas actitudes de superprotección son evidentes en los padres, pero sobretodo en los abuelos cuando asumen el papel de cuidadores principales de estos jóvenes.

Se evidenció la importancia de la existencia de un sistema de soporte adecuado, principalmente la presencia de una persona de referencia que apoye al joven y a su familia en los momentos de crisis, dando preferencia a un profesional de salud o de educación.

El modelo de referencia con EB constituye un factor clave para facilitar el proceso de autonomía, el hecho de haber vivenciado las mismas dificultades que estos jóvenes, puede proporcionar mejores estrategias para superar estos obstáculos y constituir un modelo para estos jóvenes.

Las barreras arquitectónicas fueron señaladas no sólo por los jóvenes sino también por sus cuidadores, como factores que dificultan la autonomía en las AVD's. A pesar de la actual legislación portuguesa ser clara y favorable en esta área, aún tenemos un largo camino que recorrer en lo que se refiere a la supresión de las barreras físicas. Urge que los responsables del derecho, cumplan y hagan cumplir las leyes nacionales para crear condiciones para que los niños con movilidad reducida tengan derecho a una vida lo más digna y normal posible como cualquier otro.

El asco que estos jóvenes sienten al tocar su cuerpo fue un hallazgo poco evidenciado en la literatura actual, y es una preocupación para los cuidadores en este proceso de construcción de la autonomía, puede estar relacionada con la ausencia de sensibilidad que presentan en los miembros inferiores y el modo como perciben su cuerpo y su imagen corporal ${ }^{17}$. Situación que nos alerta de la urgente necesidad de intervenir precozmente en la estimulación sensorial y percepción corporal.

Se considera que la enfermería puede asumir un papel muy importante y contribuir a la resolución de algunas de las dificultades presentadas por los participantes. A través de la identificación de las necesidades de estos jóvenes en sus contextos reales, especialmente en las escuelas, en el domicilio, podrá intervir adecuadamente no sólo a nivel escolar a través de una preparación previa y oportuna de la integración de 
estos jóvenes en la escuela, como también a nivel de la comunidad a través de programas de sensibilización para la diferencia.

Con la realización de este estudio podemos concluir que el enfermero puede desempeñar un papel crucial en la construcción de la autonomía de los jóvenes portadores de EB, en la medida en que debe actuar en una perspectiva global, abarcando las diferentes dimensiones que influyen en el nivel de funcionalidad del joven. El concepto de intervención precoz surge como respuesta a esta problemática pues la promoción de la funcionalidad en estos jóvenes servirá para prevenir problemas de salud que más tarde pueden limitar su calidad de vida, como el autosondaje, que va a prevenir las infecciones urinarias y las repercusiones nefastas del funcionamiento renal.

En un futuro, consideramos que sería pertinente la realización del mismo tipo de estudio a los profesionales que contacten diariamente con jóvenes portadores de EB, sobre todo los profesionales de salud y los de educación para que de algún modo podamos percibir si hay similitud en lo que se refiere a la opinión de los profesionales de salud y educación sobre el proceso de construcción de la autonomía de estos jóvenes con EB y así poder adecuar nuestra intervención a sus necesidades reales Sería, también interesante la realización de un estudio cuyo criterio de inclusión sería jóvenes con EB autónomos en la realización de las AVD y percibir las estrategias y experiencias que permiten que este proceso tuviese éxito.

Sugerimos, por último, la planificación e implementación de programas de promoción de la autonomía a nivel de las AVD's en la comunidad, basados en los resultados obtenidos en este estudio.

Al realizar este estudio, acreditamos y partimos del presupuesto de que todos los jóvenes tienen derecho a expresar libremente su opinión acerca de las cuestiones que les hacen al respecto y que esa opinión debe ser tenida siempre en consideración. Solo con una visión atenta y dedicada de todos estos aspectos por parte de la sociedad en general, y, en especial, de cada uno de nosotros, podremos proporcionar la calidad de vida y las sonrisas que estos jóvenes tanto merecen.

\section{BIBLIOGRAFÍA}

1. Antolovich, Giuliana C.; WRAY, Alison C. - Habilitation of Children and Young Adults with Spina Bifida. In: ÖZEK, M. Memet; CINALLI, Giuseppe; MAIXNER, Wirginia J. (coord.) - The Spina Bifida - Management and Outcome. Italia: Springer- Verlag, 2008. p.341-348. ISBN 978-88-470-0650-8.

2. Spennato, Pietro; Savarese, Luciano; Cinalli, Giuseppe - Intellectual Outcome in Spina Bifida. In: ÖZEK, M. Memet; CINALLI, Giuseppe; MAIXNER, Wirginia J. (coord.) - The Spina Bifida - Management and Outcome. Italia: Springer-Verlag, 2008. p.405-411. ISBN 978-88-470-0650-8.

3. Malheiro, Maria I. D. C. - Integração/Inclusão de Jovens com Spina Bífida no Ensino Regular... A Voz da Experiência. Lisboa: Faculdade de Motricidade Humana, Dissertação com vista à obtenção do grau de Mestre em Educação Especial, 2005.

4. Sandler, Adrian - Living With Spina Bifida - A Guide for Families and Professionals. USA: The University of North Carolina Press, 2004. ISBN 978-08078-5547-8. 
5. Barros, L. - Psicologia Pediátrica: Perspectiva desenvolvimentista (2ª Edição ed.). Lisboa: Climepsi Editores. (2003).

6. Easton, J.K.; Rens, B.; Alexander, M.A. - 6. Psychosocial Aspects of Childhood Disabilities. In: Molnar, Gabriella E.; Alexander, M. A. - Pediatric Rehabilitation. Elsevier Health Sciences (Hanley and Belfus), 1998. P. 111-124. ISBN: 978-14398-0842-9.

7. Garcia, Daniel Lerma, Medina, Diaz Carmen e Saniger, Marcelina Arrazola. Espina Bífida: Una malformación congénita. In: Revista ROL de Enfermería. ISSN 0210-5020. - Vol. 25, no 6 (Junio 2002), p. 58-64.

8. Pistrang, Nancy; Barker, Chris; Humphreys, Keith - Mutual Help Groups for Mental Health Problems: A Review of Effectiveness Studies.In: American Journal of Community Psychology. ISSN 0091-0562. Vol. 42 (2008). p. 110-121. Acedidoem 21/06/2010, de E-Journals database. Disponível em: http://search.ebscohost.com/login.aspx?direct=true\&db=eoah\&AN=15261920\&site =ehost-live.

9. Krueger, Richard A. - Analyzing \& Reporting Focus Group Results.- Focus Group Kit 6. Thousand Oaks: Sage Publications, 1998b. ISBN 0-7619-0819-6.

10.Morgan, David L. - The Focus Group Guidebook - Focus Group Kit 1. United States of America: Sage Publications, 1998. ISBN 0-7619-0760-2;

11.Morgan, David L. - The Focus Group Guidebook - Focus Group Kit 1. United States of America: Sage Publications, 1998. ISBN 0-7619-0760-2;

12.Graue, M. E., \& Walsh, D. J.Investigação etnográfica com crianças: teorias, métodos e ética. Lisboa: Fundação Calouste Gulbenkian de Lisboa. (1998)

13.Proot, Ireen M. etal. - Suporting Stroke Patients' Autonomy During Reabilitation. In Nursing Ethics. Vol. 14, no 2 (2007). ISSN: 0969-7330. pp. 229-241;

14.Lutkenhoff, Marlene, R.N., M.S.N. - Children with Spina Bifida: A Parents' Guide. Maryland: Woodbine House, 1999. ISBN: 0-933149-60-3.

15.Holmebeck, G. N., Johnson, S. Z., Wills, K., Mckernon, W., Rolewick, S., \& Skubic, T. (2002). Observed and perceived parental overprotection in relation to psychosocial adjustment in pre-adolescents with a physical disability: The mediational role of behavioral autonomy. Journal of Consulting and Clinical Psychology, 70, 96-110. Disponível em: http://www.luc.edu/psychology/pdfs/gh5.pdf

16. Hoeman, Shirley P. - Enfermagem de Reabilitação - Aplicação e Processo. $2^{a}$ Edição. Loures, Lusociência, 2000. ISBN 972-8383-13-4.

17.Rodrigues, D. Corpo, espaço e movimento: A representação espacial do corpo em crianças com paralisia cerebral. Lisboa: Instituto Nacional de Investigação Científica. (2000)

ISSN 1695-6141

๑ COPYRIGHT Servicio de Publicaciones - Universidad de Murcia 